\title{
A Review of Cosmic Rays of LHAASO
}

\section{Zhang Shoushan ${ }^{a, b}$}

${ }^{a}$ The Institute of High Energy Physics of the Chinese Academy of Sciences, YuQuan Road.19B, Shijingshan district, Beijing, China

${ }^{b}$ TIANFU Cosmic Ray Research Center, Chengdu, Sichuan, China

E-mail: zhangsseihep.ac.cn

Large High-Altitude Air Shower Observatory (LHAASO) has one square kilometer array of scintillator detectors and muon detectors, 18 Imaging Atmospheric Cherenkov telescopes and a 78,000 square meter Water Cherenkov Detector Array. LHAASO located at very high altitude (around $4410 \mathrm{~m}$ a.s.1.) in China. Multi-parameter observation of showers allows to measurement the energy spectrum, elemental composition and anisotropy with high resolution, which give us an excellent opportunity to understand the origin, acceleration and propagation of ultra-high energy cosmic rays. The $1 / 4$, the $1 / 2$, the $3 / 4$ LHAASO array have started running in September 2019, in January 2020, in December 2020 respectively. A preliminary result of absolute energy scale and the prospect of the energy spectrum, elemental composition of LHAASO experiment will be reviewed in the paper. The method of calibrating the ground-based detector with the displacement of Moon shadow is preliminarily established by LHAASO. Combined with the shower information measured by the three type of detectors of LHAASO, the pure proton energy spectrum will be obtained event by event. Accurate measurement of the position of the cosmic proton knee is very important to reveal the acceleration mechanism of ultra-high cosmic rays. This will be achieved by LHAASO in the near future.

$37^{\text {th }}$ International Cosmic Ray Conference (ICRC 2021)

July 12th - 23rd, 2021

Online - Berlin, Germany 


\section{Introduction}

Cosmic rays have been discovered for more than 100 years, but the source of the ultra-high energy cosmic rays has not been solved due to most of cosmic rays are charged particles. Gamma rays and neutrinos are uncharged particles that point to the source, which can be used to explore the origin of the ultra-high energy cosmic rays indirectly. Twelve Pevatrons gamma ray sources were discovered by LHAASO recently [1], but discriminating electron Pevatrons from cosmic ray Pevatrons is not easy. This raises a debate whether ultra-high energy gamma rays are produced by ultra-high energy electron radiation or by the interaction between ultra-high energy protons or nuclei and ambient matter around the gamma ray source. Accurate measurements of cosmic ray energy spectrum, compositions and anisotropy for different composition are also an important tool to investigate the origin, the acceleration and the propagation of cosmic rays [2]. For example, the position of the knee of cosmic rays represents the highest energy that the Galactic cosmic ray sources can reach in accelerating particles and the position of the knee for different compositions can reveal the acceleration mechanism related to rigidity or not. The $\mathrm{EeV}$ cosmic rays anisotropy detected by AUGER indicates that the EeV cosmic rays are produced in extragalactic sources [3].

Cosmic rays of energies up to about $100 \mathrm{TeV}$ can be measured by the satellite or balloon based experiments with high precision. For direct measurement experiments, classification of elements is not difficult, because they have e.g. charge-sensitive detectors. Direct measurement experiments have a small sensitive area due to limited pay-load, so that ground-based instrumentation is necessary at higher energies. Cosmic rays interacting with the atoms in the atmosphere generates a cascade which contains a large number of secondary particles, known as Extensive Air Shower (EAS). These particles are electrons, positrons, muons, hadrons, gamma rays, neutrinos, Cherenkov light and fluorescence light. Different kind of detectors on ground are design to measure these particles. The energy and composition of the cosmic ray can be obtained by measuring the information of secondary particles from EAS.

One of the major challenges of ground-based experiment is to classify single element event by event. By using unfolding analysis techniques, the spectra of five primary mass groups (represented by $\mathrm{H}, \mathrm{He}, \mathrm{CNO}, \mathrm{MgSi}, \mathrm{Fe}$ ) are derived by KASCADE experiment [6] from the electromagnetic components and muon components in EAS. However, the spectra of five primary mass groups are not resolved event by event. The ARGO-YBJ experiment and a image air Cherenkov telescope have made an important step in the event by event composition classification. The $\mathrm{H}+\mathrm{He}$ spectra from $100 \mathrm{TeV}$ to $3 \mathrm{PeV}$ are resolved event by event, showing a knee like structure at $700 \mathrm{TeV}$. The Large High-Altitude Air Shower Observatory (LHAASO) experiment has electromagnetic detectors, muon detectors, water Cherenkov detectors and image air Cherenkov telescopes, which allows LHAASO to measure multi-component information of EAS. LHAASO is expected to resolve the individual composition spectra by using event by event method. Another major challenge of ground-based experiment is absolute energy scale calibration. The displacement of the moon shadow caused by the bending of charged cosmic rays in the geo-magnetic field, was measured by the ARGO-YBJ experiment [5]. The ARGO-YBJ experiment obtains an absolute energy scale with an uncertainty of $12 \%$ for the first time by using the displacement as a function of cosmic ray energy. The absolute energy scale can be also obtained by the LHAASO experiment by using the same method. A preliminary result of absolute energy scale and the prospect of the energy 
spectrum, elemental composition of LHAASO experiment will be reviewed in the paper.

\section{LHAASO experiment}

The LHAASO experiment [1] located at Haizishan mountain, Sichuang province, China, with an altitude of $4410 \mathrm{~m}$ above see level. It consists of a $1 \mathrm{~km}^{2}$ EAS array (KM2A) [8], a water Cerenkov detector array (WCDA) [9], a wide field of view Cherenkov telescope array (WFCTA) [10]. KM2A includes 5195 scintillator detectors, with $15 \mathrm{~m}$ spacing, for electromagnetic particle detection and 1188 underground water Cherenkov tanks ( $36 \mathrm{~m}^{2}$ per tank), with $30 \mathrm{~m}$ spacing, for muon detection. WCDA has two $150 \mathrm{~m} \times 150 \mathrm{~m}$ water pools plus one $300 \mathrm{~m} \times 110 \mathrm{~m}$ pool, namely WCDA01, WCDA02 and WCDA03 respectively. WCDA has total area of about 78,000 $\mathrm{m}^{2}$ and 3120 cells, and each cell is $5 \mathrm{~m} \times 5 \mathrm{~m}$ in size. Two adjacent PMTs are installed in each cell. A one-inch PMT and a eight-inch PMT are used for each cell for WCDA01, and a three-inch PMT and a twenty-inch PMT are used for each cell for WCDA02 and WCDA03.

WFCTA has 18 Cherenkov telescopes. Each Cherenkov telescope consists of an array of $32 \times 32$ SiPMs and a $\sim 5 \mathrm{~m}^{2}$ spherical aluminized mirror. It has a field of view (FOV) of $16^{\circ} \times 16^{\circ}$ with a pixel size of approximately $0.5^{\circ} \times 0.5^{\circ}$. In the first observation stage, the main axis of six telescopes are arranged to point at the zenith angle of $30^{\circ}$, and cover $22^{\circ}$ to $38^{\circ}$ in zenith and $0^{\circ}$ to $156^{\circ}$ in azimuth. WFCTA, WCDA01 and muon detectors aim to measure the energy spectra of proton and light component around the first knee in the first observation stage. In the second observation stage, the main axis of eighteen telescopes are arranged to point at the zenith angle of $45^{\circ}$, and cover $37^{\circ}$ to $53^{\circ}$ in zenith and $0^{\circ}$ to $360^{\circ}$ in azimuth. WFCTA and KM2A aim to measure the energy spectra of iron and heavy component around the second knee in the second observation stage.

The first six telescopes which lactated at the the southwest corner of the first pond of WCDA, started operation in October 2019. Six telescopes are pointed to zenith angle of $60^{\circ}$ and cover the azimuth angle of $0^{\circ}$ to $156^{\circ}$. Two of the remaining twelve telescopes operation in January 2020 and ten in April 2021, which located at the southeast corner of the first pond. The first six telescopes were moved to the southeast corner of the first pond and total eighteen telescopes are pointed to zenith angle of $45^{\circ}$ and cover the azimuth angle of $0^{\circ}$ to $360^{\circ}$ in May 2021. The $1 / 4 \mathrm{KM} 2 \mathrm{~A}$ and WCDA01, the 1/2 KM2A and WCDA02, the 3/4 KM2A and WCDA03 have started running in September 2019, in January 2020, in December 2020 respectively. The layout of eighteen WFCTA telescopes is shown in Fig. 1.

\section{A preliminary results of absolute energy scale}

In the direct measurement experiments, such as CREAM, a balloon-borne experiment, the detectors were calibrated pre-flight at the European Organization for Nuclear Research (CERN) using the available highest-energy proton and electron test beams [4]. The beam of known energy is a dream of ground-based experiments. The charged cosmic rays are deflected by the geo-magneticfield (GMF) before reaching the detectors on the ground. The deficit of the cosmic rays blocked by the Moon, the Moon shadow of cosmic rays, is measured by the detectors. Obviously, the Moon shadow can shift respect to the Moon real position due to the charged cosmic rays bending in GMF. The displacement is as a function of cosmic ray energy and is clearly dependent on the cosmic ray 


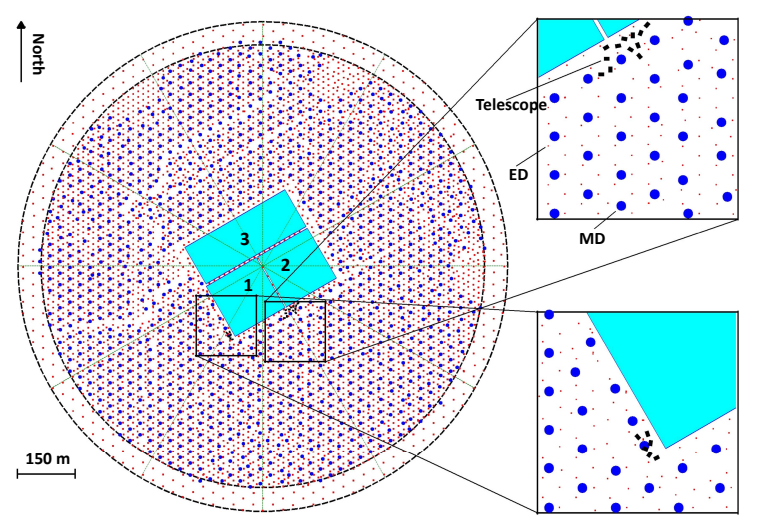

Figure 1: The layout of eighteen LHAASO-WFCTA telescopes before April 15, 2021 [10]. The mall black rectangles indicate the telescopes. Six telescopes are located at the southwest corner of WCDA01, and are pointed to zenith angle of $60^{\circ}$ and cover the azimuth angle of $0^{\circ}$ to $156^{\circ}$. The six telescopes were moved to the southeast corner of WCDA01 in May 2021, and then total eighteen telescopes are pointed to zenith angle of $45^{\circ}$ and cover the azimuth angle of $0^{\circ}$ to $360^{\circ}$. Two big squares and a big rectangle with numbers in the center of LHAASO array indicate three ponds of WCDA, namely WCDA01, WCDA02, WCDA03. The blue dots indicate muon detectors and the red squares indicate scintillator detectors.

rigidity. The displacement is clearly observable at energies below $40 \mathrm{TeV}$ and becomes negligible at energies higher than $100 \mathrm{TeV}$. The charged cosmic rays skim the Moon surface, deflected by GMF and measured by the detectors, which are calibrated as the beams with known energy. The ARGO-YBJ experiment obtains an absolute energy scale for the first time by using the beams [5].

WCDA01 has a detection threshold of about $1 \mathrm{TeV}$ for cosmic rays. The data set from $01 / 05 / 2019$ to $31 / 01 / 2020$ was used. The total effective observation time is about 731.2 hours at the condition of the Moon with zenith angles $\leq 45^{\circ}$, and the total number of events is about 4.17 million at the condition of arrival direction within $5^{\circ}$ to the Moon position. First, the Moon shadow shift westward is measured as a function of the total number of photo-electron Npe measured by WCDA01, i.e., the displacement $\Delta$ vs. Npe. Second, the displacement as a function of primary cosmic ray energy is calculated by tracing the cosmic rays with certain composition through GMF. The function $\Delta=1.59^{\circ} /($ Energy $/ Z)$ is established. Here, $Z$ is the charge of nucleus. Third, the average rigidity of cosmic ray events that trigger WCDA01 in the relevant ranges of Npe is calculated by using the air shower and detector response simulation. The flux ratio between $\mathrm{H}$ and He measured by the CREAM [13] and DAMPE [12] experiment in this energy range are taken into account. Accordingly, the displacement $\Delta=2.1 / \mathrm{E}[\mathrm{TeV}]$ is obtained, where $\mathrm{E}$ is the median energy of the cosmic beam in the relevant ranges of Npe. After integrating the above three steps, the average shower energy calculated using displacement of the Moon shadow measured by WCDA01 versus the total number of photo-electrons Npe measured by WCDA01 is established and is shown in Fig.2. And then, the function $E[\mathrm{GeV}]=1.33 \cdot N_{p e}^{0.95}$ is established. The criteria, the number of fired cells $\geq 200,20,000 \leq N p e \leq 60,000$ and $22^{\circ} \leq$ zenith angle $\leq 38^{\circ}$, are used. The value obtained for the shift in the criteria is $\Delta=(0.10 \pm 0.03)^{\circ}$. And then the energy scale of $21.0 \pm 6.5$ $\mathrm{TeV}$ are obtained by using the formula of $\Delta=2.1 / \mathrm{E}[\mathrm{TeV}]$ as before. After the further selection the shower core falling inside WCDA01, the energy scale of $16.2 \pm 6.2 \mathrm{TeV}$ is obtained. The uncertainty 
of the energy scale is largely dominated by the low statistics of events, which will be improved by accumulating longer observation time in future. The total uncertainty varies from $12 \%$ at 6.6 $\mathrm{TeV}$ to $50 \%$ at $35 \mathrm{TeV}$. The uncertainty caused by different composition models is about $3 \%$. The uncertainty caused by different hadronic models is less than $2 \%$. The uncertainty caused by the energy resolution and angular resolution is about $4 \%$.

Although WFCTA can work on Moon nights, the intensity of direct moonlight is enough to saturate the telescope. Thus, the Moon shadow can not be observed by WFCTA. Therefore, a set of commonly triggered events of WFCTA and WCDA01 is used to propagate the absolute energy scale of WCDA01 to WFCTA. And 10 nights in Jan. 2020 with moonless and very excellent weather conditions are selected. In order to achieve a more precise energy reconstruction, the shower cores are required to locate inside the WCDA01 and the full Cherekov image are measured by applying telescope FOV cut. Reconstructed energies distribution of commonly triggered events of WCDA01 and WFCTA is shown in Fig.3. The energy distribution of WCDA01 is calculated by using the formula $E[\mathrm{GeV}]=1.33 \cdot N_{p e}^{0.95}$ as before. The median energy of the distribution is $(23.4 \pm 0.1 \pm 1.3) \mathrm{TeV}$. The median energy measured and reconstructed by WFCTA is $21.9 \pm 0.1$ $\mathrm{TeV}$. The energy scale of WCDA01 is propagated to WFCTA by a set of commonly triggered events. This is the first time the Cherenkov telescopes have the absolute energy scale. More details of the preliminary results of absolute energy scale can be seen in [11].

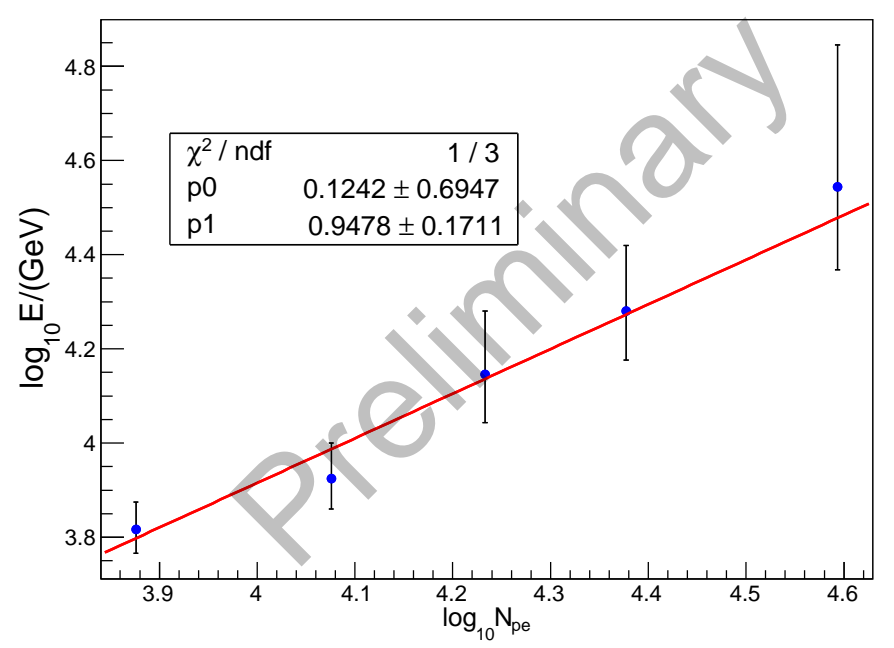

Figure 2: The average shower energy calculated using displacement of the Moon shadow measured by WCDA01 versus the total number of photo-electrons Npe measured by WCDA01 [11].

\section{The prospect of cosmic rays energy spectrum and composition}

The shower geometry are well measured by WCDA, and the resolution of shower core is about $3 \mathrm{~m}$ and the angular resolution is about $0.2^{\circ}$ above $10 \mathrm{TeV}$ [9]. The shower core resolution and angular resolution of is about $3 \mathrm{~m}$ and less than $0.3^{\circ}$ above $100 \mathrm{TeV}[8]$. The number of muon in the air shower is a powerful parameter to discriminate cosmic compositions. The detection area of LHAASO muon detectors is about $42,000 \mathrm{~m}^{2}$, and that of KASKADE is about $600 \mathrm{~m}^{2}$. Because 


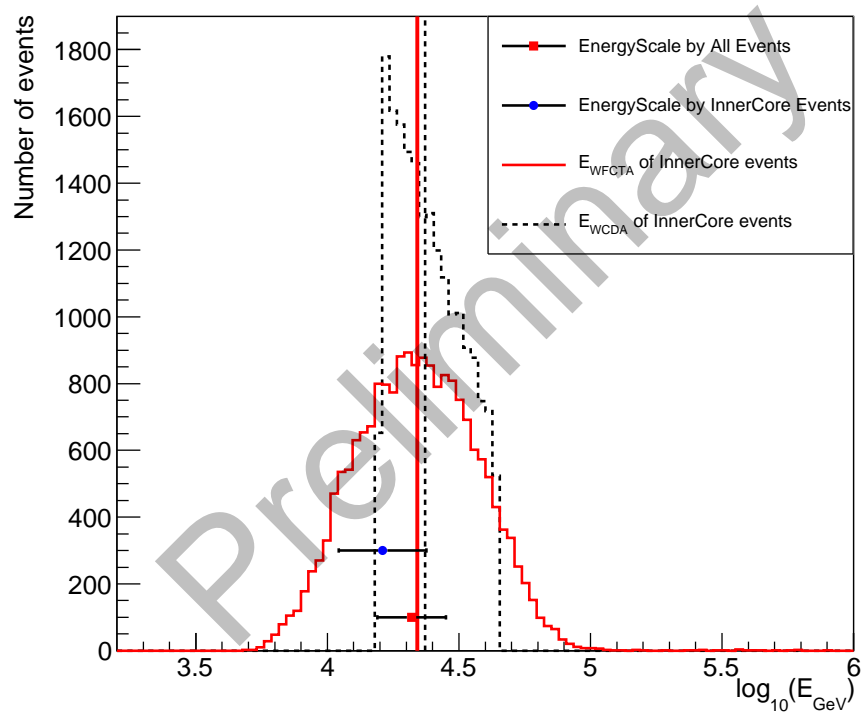

Figure 3: Reconstructed energies distribution of commonly triggered events of WCDA01 and WFCTA [11]. The red solid line is the reconstructed energy distribution of the WFCTA telescopes. The median energy of the distribution (vertical red solid line) is $(21.9 \pm 0.1) \mathrm{TeV}$. The black dashed line is the energy distribution of WCDA01, which is calculated by using the formula $E[\mathrm{GeV}]=1.33 \cdot N_{p e}^{0.95}$. The median energy of the distribution (vertical black dashed) is $(23.4 \pm 0.1 \pm 1.3) \mathrm{TeV}$. The median energies $21.0 \pm 6.5 \mathrm{TeV}$ (red square) obtained from the Moon shadow data is related to the selection Nhit $>200,20,000<\mathrm{Npe}<60,000,22^{\circ}<$ zenith angle $<38^{\circ}$. After the further selection the shower core falling inside WCDA01, the median energy becomes $16.2 \pm 6.2 \mathrm{TeV}$ (blue dot), which is largely dominated by the low statistics of events.

of the secondary particles induced by heavier nuclei in EAS are more diffused to the farther area laterally and produce a more uniform distribution. The energy spectrum of cosmic protons and helium nuclei below $100 \mathrm{TeV}$ can be measured by WCDA01. The spectrum below $100 \mathrm{TeV}$ will overlap and cross check with the cosmic rays energy spectrum of the direct experiment. There are significant differences between the lateral distributions of EAS induced by light and heavy nuclei. The number of particles near the shower core and extension rate of the shower lateral distribution are two composition sensitive parameters, which can be measured by WCDA. Cherenkov images measured by Cherenkov telescopes of LHAASO look like an ellipse and can be described by Hillas parameters [14]; such as the width and length of the image. The width is related to the lateral development of the shower and the length is related to the longitudinal development of the shower. Therefore, they are two good parameters that is sensitive to the primary composition. In fact, the angular profile of number of photo-electrons along the length direction is related to the longitudinal development of Cherenkov photons in EAS, as shown in Fig. 4. The Cherenkov image accumulates photons produced by shower charged particles at every stage of the shower's longitudinal development. Therefore, the total number of photo-electrons in the Cherenkov image together with the shower geometry precisely measured by KM2A or WCDA, can reconstruct the shower energy precisely. The energy resolution is found to be about $15 \%$ for proton with bias less than 2\%. The Xmax can be obtained from the angular profile and the resolution of Xmax is found to be about $40 \mathrm{~g} / \mathrm{cm}^{2}$. More details can be seen in [16].

The data set from November 5, 2020 to March 3, 2021 are counted. WCDA01 and WCDA02, 
$1 / 2 \mathrm{KM} 2 \mathrm{~A}$, and six telescopes are in stable operation during this time. After the selection of good weather and the stable operation of three type of detectors, the effective hybrid observation time is 750 hours. The energy spectrum of cosmic protons will be analyzed by a combination of the shower information measured by three type of detectors. About 50 events of pure proton is expected to be measured at energy around $3 \mathrm{PeV}$ by assuming the proton keen at $700 \mathrm{TeV}$ which is claimed by the ARGO-YBJ experiment and WFCTA prototype [15].

WCDA is a full coverage detector and the energy threshold for proton and helium is about 1 $\mathrm{TeV}$. The energy spectrum of cosmic protons and helium nuclei below $100 \mathrm{TeV}$ can be measured by WCDA01. This give the ground-based experiment to have the opportunity to measure the cosmic ray spectrum in a common energy range together with direct measurements and to compare results with direct measurements.
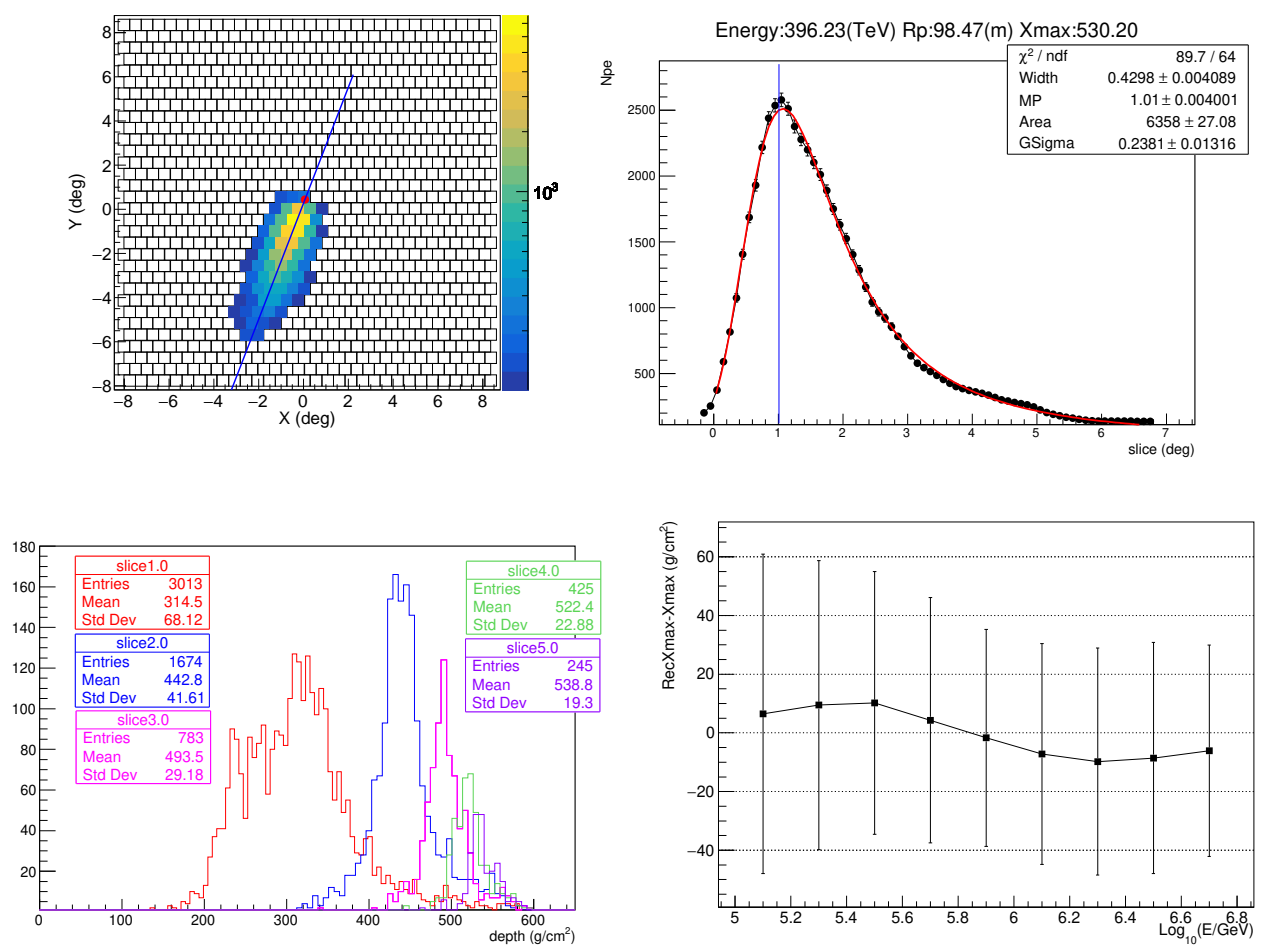

Figure 4: Along the length direction of Cherenkov image (up left) measured by WFCTA from simulation, the image is segmented into several slices, each with a step length of $0.5^{\circ}$. The number of photo-electrons in each slice as a function of angular distance is shown, namely image angular distribution, and it can be fitted well by a function convolving the Landau distribution with Gaussian distribution (up right). The peak of the image angular distribution is around $1.0^{\circ}$ which corresponds to the average atmosphere depth of 314 $\mathrm{g} / \mathrm{cm}^{2}$. The number of photons as a function of atmosphere depth in the slices of $1.0^{\circ}, 2.0^{\circ}, 3.0^{\circ}, 4.0^{\circ}$ and $5.0^{\circ}$ are shown from left to right respectively (down left). Cherenkov image accumulates photons produced by shower charged particles at every stage of the shower's longitudinal development. The width of the image angular distribution is related to the $X_{\max }$ and the resolution of $X_{\max }$ over cosmic rays energies is shown (down right). The resolution of $X_{\max }$ is about $40 \mathrm{~g} / \mathrm{cm}^{2}$. [16] 


\section{Conclusion and discussion}

The method of calibrating the ground-based detector with the displacement of Moon shadow is established by LHAASO. The absolute energy scale are obtained by WCDA01 preliminarily and propagate from WCDA to WFCTA by using the common trigger events. The Cherenkov image accumulates photons produced by charged particles in every stage of shower longitudinal development. So, the shower energy can be measured well by WFCTA. Combined with the shower information measured by the three type of detectors of LHAASO, the pure proton energy spectrum will be obtained event by event. During the operation period from November 5, 2020 to March 3, 2021, assuming that proton knee is around $700 \mathrm{TeV}$, it is estimated that about 50 pure proton events will be measured by LHAASO at a primary energy of $3 \mathrm{PeV}$. Accurate measurement of the position of the cosmic proton knee is very important to reveal the acceleration mechanism of ultra-high cosmic rays. This will be achieved by LHAASO in the near future.

\section{References}

[1] Cao, Z., Aharonian, F.A., An, Q. et al., Nature 594, 33-36 (2021)

[2] E. Berezhko, S. Knurenko, L. Ksenofontov, Astroparticle Physics, vol. 36(1), (2012)

[3] V. Berezinsky, A. Gazizov, S. Grigorieva, Phys. Rev. D, vol. 74, (2006) p. 043005

[4] H. S. Ahn et al., The Astrophysical Journal Letters, 714:L89-L93, 2010

[5] B. Bartoli, Physical Review D 84, 022003 (2011)

[6] T. Antoni et al., Astroparticle Physics 24 (2005) 1-25

[7] Q. An et al.(for LHAASO Coll.), Nucl.Instrum.Meth. A, 2013, 724: 12-19.

[8] F. Aharonian et al., 2021 Chinese Phys. C 45025002

[9] F. Aharonianet et al., arXiv:2101.03508v1 [astro-ph.IM]

[10] F. Aharonianar et al., Xiv:2012.14622 [physics.ins-det], 2020

[11] F. Aharonianet et al., arXiv:2104.04965 [astro-ph.IM]

[12] Q.An,et al.,Science Advances 5 (9) (2019) eaax3793

[13] Y.S.Yoon, et al., Astrophysical J., 839:5 (2017)

[14] Hillas, A. 1985, 19th ICRC V3, 445-448.

[15] B. Bartoli et al., PHYSICAL REVIEW D 92, 092005 (2015)

[16] Ma Lingling et. al., "Measurement of the cosmic ray proton and helium spectrum with energy above $100 \mathrm{TeV}$ by the LHAASO experiment", proceeding of 37th ICRC (2021). 